\title{
MODELANDO A INTEGRALIDADE DO CUIDADO À CRIANÇA NA ATENÇÃO BÁSICA DE SAÚDEa
}

F rancisca G eorgina M acedo de SOUSA ${ }^{b}$, A lacoque Lorenzini ERD M AN N c, Elba Gomide M OCHE L ${ }^{b}$

\section{RESUM 0}

Cuidar da criança na Atenção Básica de Saúde (ABS) na perspectiva da integralidade sugere organização e abordagem envolvendo articulação de saberes e fazeres. N esse sentido, questiona-se: Que práticas, gestores, formadores e profissionais da saúde reconhecem como necessárias para a integralidade do cuidado? 0 estudo teve como objetivo compreender como são produzidas as práticas de cuidado à criança na atenção básica na perspectiva da integ ral idade em saúde. 0 s pressupostos da abordagem qual itativa e da G rounded Theory guiaram a coleta e análise dos dados aliada à entrevista semi-estruturada. Participaram da investigação 29 sujeitos organizados em cinco grupos amostrais. Descreve-se uma das categorias construídas na investigação nomeada "M odelando a Integralidade do Cuidado à Criança na ABS". Esse conceito é caracterizado pelo modo de encontro e preparo dos profissionais para uma aproximação com a família por meio do trabal ho em equipe e práticas intersetoriais, aliando ao saber técnico as tecnologias relacionais e subjetivas.

D escritores: Enfermagem pediátrica. Cuidado da criança. A tenção primária à saúde.

\section{RESUMEN}

Cuidar del niño en la Atención Básica de Salud en la perspectiva de la integralidad sugiere una organización y un abordaje incluyendo una red articulada de saberes y hechos. E $n$ ese sentido preguntamos: ¿Qué prácticas de gestión profesional y de las madres son reconocidas como necesarias para la integralidad del cuidado? T uvo como objetivo comprender como son producidas las prácticas del cuidado al niño en la atención básica. L os presupuestos del abordaje cualitativo y de la G rounded Theory guiaron la recolección y análisis de los datos, unida a la entrevista semi-estructurada. Participaron de la investigación 29 personas. Se describe uno de los conceptos macros construido en la investigación denominado M odelando la Integralidad del Cuidado al niño en la A tención B ásica de Salud. E ste concepto se caracteriza por el modo de encuentro y preparación de los profesionales para una aproximación con la familia por medio del trabajo en equi po y delas prácticas inter sectoriales asociando al saber técnico las tecnologías relacional es y subjetivas.

Descriptores: E nfermería pediátrica. Cuidado del niño. A tención primaria de salud.

Título: M odelando la integralidad de los cuidados a los niños en la A tención B ásica de la Salud.

\section{ABST RACT}

Taking care of children in Basic $\mathrm{H}$ ealth Attention in completeness perspective, suggests organization and broach involving an articulated net of "knowing" and "doing". To this end, an inquiry was madeby theauthors of this study, the N ursing re searchers: W hich practices, administrators, professionals and mothers recognize as necessary for the completeness of taking care? The investigation aims to comprehend how the practices of taking care of children under basic attention are produced according to the completeness perspective $T$ he prer equisites of the qualitative broach and the $G$ rounded $T$ heor $y$ guided the data surveying and anal ysis along with a semi-structured interview. T w enty-nine peopleorganized into five groups participated in the research. 0 ne of the concepts built during the research is described and nominated as the $M$ oulding of Completeness Taking Care of Children in Basic $\mathrm{H}$ ealth Attention. This concept is characterized by the meeting and preparation of the professionals for an approach with the families through a team work and for intersectoral practices allying the technical knowledge to the related and subjected technologies.

D escriptors: Pediatric nursing. Child care. P rimary health care.

Title: M olding the integration of children care in Basic $\mathrm{H}$ ealth Attention.

\footnotetext{
a Extraído da tese de D outorado apresentada em 2008 ao Programa de Pós-G raduação em Enfer magem da U niversidade Federal de Santa Catarina (U FSC).

${ }^{b}$ D outora em E nfer magem, D ocente do D epartamento de E nfermagem da U niversidade F ederal do M aranhão (U F M A ), São L uís, M aranhão, Brasil.

'D outora em E nfer magem, D ocente do Programa de Pós-G raduação em Enfermagem da U F SC, Pesquisadora 1A do CN Pq, F lorianópolis, Santa Catarina, Brasil.
} 


\section{INT RODUÇÃO}

0 cuidado à criança é complexo, multidimensional sofrendo influência de diversos fenômenos das ações do cuidar. A integralidade, como princípio da política de saúde, remete à compreensão de que os fatores que interferem na saúde da criança são amplos e perpassam por outros setores que não só a saúde. Assim, a integralidade é sustentada e defendida como valor nas práticas dos profissionais de saúde que se expressa na forma como estes respondem às necessidades das pessoas atendidas(1). Portanto, a concretização de um sistema integral de saúde não passa pela aplicação exclusiva dos saberes disciplinares(2), mas é entendida como "o entre-relações de pessoas, com efeitos e repercussões de interações positivas entre usuários, profissionais e instituições traduzidas em tratamento digno e respeitoso, com qualidade, acolhimento e vínculo"(3). N essa medida, 0 cuidado será possível se há condições concretas para o diálogo, para o vínculo e para a solidariedade.

Especificamente no que diz respeito ao cuidado à criança na Atenção Básica de Saúde (ABS) a proposta dirige-se para organização dos serviços aliado a um processo que envolve uma rede de instituições e serviços de forma a obter impacto sobre as diversas circunstâncias do processo de viver da criança. T rata-se de um fazer/ pensar integrado do serviço e da equipe e não da tarefa de um profissional ou de uma categoria isoladamente. $M$ as, o modelo médico hegemônico ainda prevalece nas práticas em saúde e se expressam por ações prioritariamente curativas, centradas na doença e sem a complementaridade das ações de proteção, promoção e prevenção(4) de agravos à saúde infantil. Perde-se assim, a oportunidade de um olhar ampliado para as necessidades do crescimento e desenvolvimento infantil, compartilhadas com aquelas inerentes à família e ao ambiente no qual a criança está inserida. A poiada nestas assertivas questiona-se: Que práticas gestores, docentes e profissionais da saúde reconhecem como necessárias para a integralidade do cuidado? 0 estudo teve como objetivo compreender como são produzidas as práticas de cuidado à criança na $A B S$ na perspectiva da integralidade a partir dos significados atribuídos por gestores, coordenadores e chefes de serviços da ABS, docentes, enfermeiros e médicos da Estratégia Saúde da Família.

\section{METODOLOGIA}

Estudo qualitativo apoiado na Grounded Theor $\mathrm{y}^{(5,6)}$. Participaram do estudo 29 sujeitos entre gestores da saúde, docentes de pós-graduação e graduação em Enfermagem e M edicina, enfermeiros e médicos das Equipes de Saúde da Família de São Luís, M aranhão. Os sujeitos de pesquisa foram organizados em cinco grupos amostrais utilizando o recurso da amostragem teórica para assim garantir o refinamento e densidade das categorias. Como o objeto investigado encontra-se situado sob as dimensões da política de saúde, da organização do sistema de saúde e das práticas de cuidado, procurou-se buscar, nestas três dimensões, os participantes da investigação.

Participaram do primeiro grupo amostral os gestores estadual e municipal de saúde, os coordenadores e chefes de serviços da ABS totalizando sete participantes. 0 segundo grupo amostral foi composto por coordenadores de curso de graduação (Enfermagem e M edicina); coordenadores de pós-graduação (E specialização em Saúde da Família e M estrado em Saúde da M ulher e da Criança) e docentes da graduação, total izando seis participantes. 0 terceiro grupo amostral envolveu sete profissionais do PSF (cinco enfermeiros e dois médicos). Para este grupo foram definidos os seguintes critérios de inclusão: ser U nidade Saúde da Família, profissionais com vínculo definido por concurso público e em exercício profissional por pelos menos um ano na equipe. A partir dos critérios estabelecidos, foram selecionados profissionais de quatro U nidades de Saúde da F amília (dois na área urbana e dois na periferia da capital maranhense) em um total de quatro E quipes de Saúde da Família. 0 quarto grupo foi constituído por cinco profissionais do PSF para os quais foram estabelecidos os mesmos critérios do grupo anterior. 0 quinto grupo amostral foi composto por quatro mães, cujas famílias eram cadastradas no PSF e as crianças acompanhadas pela equipe de saúde. Participaram da pesquisa como um todo 29 sujeitos dentre os quais profissionais de 10 equipes de saúde da Família de um total de 22 existentes no município. No entanto, para a categoria e subcategorias descritas neste texto não estão incluídas as falas e os conceitos desenvolvidos com os participantes do quinto grupo amostral.

Para a coleta de dados foi utilizada a entrevista semi-estruturada realizada individualmente 
com cada participante. Durante o processo de coleta, análise e construção dos conceitos foram consideradas as etapas da codificação aberta, axial e sistemática atendendo ao princípio da amostragem teórica que tem como finalidade construção de novas hipóteses, desenvolver conceitos e refinar categorias.

N este tex to será apresentado um dos macros conceitos (categoria) e os respectivos micros conceitos (subcategorias) desenvolvidos na investigação de doutoramento em E nfermagem ${ }^{(7)}$. 0 projeto de pesquisa foi aprovado pelo Comitê de Ética do H ospital U niversitário da U niver sidade F ederal do $\mathrm{M}$ aranhão sob o registro $254 / 06$, processo no 33104-850/2006.

\section{RESULTADOS}

\section{Revelando o macro conceito: modelando a integralidade do cuidado à criança na ABS}

É um conceito que permite estabelecer correlações entre as distintas ações profissionais para a finalidade maior do cuidado que é atender a criança na sua multidimensionalidade e não somente a doença. É uma perspectiva de cuidado dirigida a problemas transversais que não podem ser tratados separadamente por uma disciplina ou por uma categoria profissional isoladamente, nem tampouco por um único serviço. Conduz ao trabalho em equipe, interdisciplinar e intersetorial. N essa dimensão se buscou desvelar a produção do cuidado como objeto do trabal ho em saúde caracterizado pelas subcategorias: encontrando integralidade do cuidado no trabal ho em equipe; enxergando o sentido interdisciplinar para o cuidado; exercitando práticas intersetoriais; valorizando a aproximação com a família; defendendo o preparo do profissional para atuar na Atenção Básica de Saúde, descritas a seguir:

\section{Encontrando integralidade do cuidado no trabalho em equipe}

É um conceito que carrega a exigência do conhecimento e valorização do trabalho do outro, construindo consensos quanto aos objetivos a serem alcançados e a maneira mais adequada de atingi-los. Requer complementaridade pela articulação de ações e conhecimentos de cada agente assim como o conhecimento acerca do trabalho do outro, como verbalizado nas falas:
A qui não existe o médico, a enfermeira, 0 auxiliar e o agente de saúde, existe a equipe (P 1).

No trabalho em equipe um profissional não tem um saber maior que 0 outro. Existe um compartilhar desses saberes num nível horizontal enão na verticalização de saberes. N ão há hierarquia, deve haver compartilhar de saberes e reconhecimento de que todos os profissionais são importantes. [ ...] é a soma dos saberes, produto do diálogo de iguais (G1).

Existe um olhar de cada profissional que deve ser valorizado. [ ...] éna soma detudo isso, que dá sentido a integralidade do cuidado (P2).

Os participantes expressaram que no cuidado à criança buscam os demais membros da equipe e de outras equipes para trocar informações, visando ao esclarecimento de dúvidas e a resolutividade dos problemas apresentados pela criança. $N$ esse sentido, a eficiência e a eficácia dos serviços na perspectiva da integralidade, requerem uma modalidade de trabal ho que traduza formas de conectar as diferentes ações e os distintos profissionais em um coletivo de agentes presentes para atender as demandas em saúde ${ }^{(8)}$, como demonstrado pelos participantes:

[ ...] a criança éatendida pelo enfermeiro ou pelo mé dico. Às vez es pelos dois no mesmo atendimento. Caso exista al gum problema com a criança que estou atendendo chamo o médico, discutimos a mel hor forma de intervenção. 0 médico vem, meajuda, tira dúvidas. T em situaç̃ões que não tenho como resolver, o que exige a presença do médico ou de outro profissional. I sso facilita. 0 trabalho fica mais fortal ecido e fico mais tranqüila, mais segura (P3).

P rocuramos essa articulação de saberes na nossa equipeenas outras equipes. Cada um tem um jeito deol har, as vez es 0 olhar de um é capaz de perceber coisas que passaram despercebidas ejá antecipa uma inter venção ou porque tenho mais habilidade em determinada ação sou chamada a intervir porque naquele momento este ja mais preparada (P4).

Os relatos apontam para existência de trocas de saberes, experiências e habilidades numa articulação entre as ações desenvolvidas pelos profissionais da mesma equipe e entre equipes. É interessante ressaltar que 0 trabal ho em equipe apresenta duas conformações: equipe agrupamento e integração(8). A primeira noção é car acterizada pela fragmentação e a segunda, pela articulação con- 
soante à proposta da integralidade das ações de saúde. A interação dos agentes permite a construção de um "projeto comum"(9) de cuidado pela comunicação e cooperação. $\mathrm{N}$ as falas dos participantes há os dois tipos de trabalho em equipe:

Existe o médico, a enfermeira, os agentes, mas trabalho integrado que éa lógica do trabal ho em equipe não existe. $\mathrm{E} u$ faço, a enfermeira faz epronto (G2).

Somos duas equipes de P SF, mas estamos tão entrosados que não parece duas pela forma como interagimos na equipe, a pesar de equi pes diferentes. T rabal hamos juntos. A queles problemas das crianças que um não pode resolver 0 outro atende eassi $m$ estamos evitando que as nossas crianças sejam encaminhadas para outros ser viços (P 5).

Trabal hando em equipe estaria relacionado com saberes profissionais que objetiva um trabaIho coletivo, buscando desafios nos modos de cuidar, de organizar e operacionalizar a gestão do cuidado.

\section{E nxergando o sentido interdisciplinar para 0 cuidado}

0 cuidado é produto e produtor de um trabalho articulado entre profissionais e destes com a família e a comunidade. É interdisciplinar enquanto prática dinâmica e processual que implica na construção de conhecimentos e na aquisição de competências ${ }^{(9)}$ em uma prática inter ativa entre as diversas disciplinas que fizeram parte das reflexões dos participantes da investigação:

[ ...] a equipe desenvolve açoes específicas do cuidado à criança, mas de forma integrada, porque não há integralidade sem um trabalho interdisciplinar, sem essa interface, sem essa integração de um saber profissional com o saber do outro. I sso é essencial. P orque tudo tem relação com tudo. A integralidade tem o sentido da interdisci plinaridade e do trabal ho em equipe, de uma ação completa e que complementa a outra (P6).

Cuidar da criança épensar nessa rede de relações, que transcende o profissional de saúde e alcança a família, a comunidade e outros profissionais, uma rede mesmo, no sentido de relaç̃oes, de entrelaçamento de objetivos, de metas, de competências, de saber es onde éimportante assumir diálogo e negociação (P2).

Portanto, as subcategorias supra citadas são caracterizadas por expressões como parceria, in- ter ação, ajuda, cooper ação, comunicação, reconhecimento de saberes e de capacidades do outro, negociação e relações que se traduz na integralidade do cuidado.

\section{Exercitando práticas intersetoriais}

$N$ essa perspectiva, o cuidado é compreendido como projeto que demanda participação cooperativa, articulação entre profissionais de diferentes áreas e instituições assim como saberes e fazeres. Os participantes reconhecem a importância do agir intersetorial procurando envolver vários organismos da sociedade civil e do Estado na resolução dos problemas da criança:

H á necessidade dessa rede articulada que responda às necessidades da popula ção infantil e de suas famílias, numa integração das ações e das institui ç̧ões. A saúde não existe por si só. $N$ ão são as unidades de saúde e os profissionais que garantem ou promovem a saúde, mas toda uma rede articulada de instituições eatores. Q uando sefala da criança deve selembrar que ela vai precisar de creche, pré escola, educação infantil, ambiente saudável, moradia, saneamento. São coisas que estão além da saúde, vão muito além (P2).

[ ...] não vou conseguir resolver todos os problemas somente com orientação profissional e com medicamentos. A integralidade compreende atender a criança vinculada ao contexto social, cultural, familiar, enxergando o sistema, a necessidade de se trabal har a intersetorialidade para assim alcançar a integralidade (G 2 ).

[ ...] uma das coisas importantes para a integralidade são as parcerias com a equipe, com os agentes, com as famílias, com a escola e com a rede. As experiências vivenciadas com Associações de M oradores e com 0 Conselho M unicipal de Saúde apresentou resultado muito positivo (P7).

N ossa função é exercitar trabalhar com o outro, expandir as ações de saúde para além do campo biológico. B uscar resolver os problemas fora da saúde, mas incl uindo a saúde, com outras insti tui ções, associações, re presentação comunitária (G2).

A intersetorialidade consiste em identificar necessidades, traçar objetivos comuns e perseguilos ${ }^{(10)}$ mediante planejamento e definição de prioridades. A importância, como revelado nas falas, é proporcionar e efetuar uma ação mais abrangente pela participação de setores e instituições que se complementam para o alcance de resultados, tor- 
nando a ação do cuidar e do cuidado mais dinâmica e produtiva.

\section{Valorizando a aproximação com a família}

Promover a saúde da criança implica agir/ intervir no contexto familiar. Esta aproximação é reconhecida pelos participantes como experiência positiva e diferenciada no cuidado à criança. É condição que permite ao profissional se deslocar para o cotidiano da família e da comunidade e, assim, optar por estratégias compatíveis com a realidade das crianças sob seus cuidados. Parece ampliar e potencializar o papel do profissional na saúde da criança por oportunizar um espaço de diálogo entre a família e o profissional favorecendo 0 relato de problemas, dificuldades e limitações no e para o cuidado. D eram suporte a esta subcategoria as falas:

$N$ a medida em que trabalhamos com as mães e com a família estamos fortal ecendo, sedimentando uma prática que é fundamental para a promoção da saúde da criança. [ ...] compreender a família como unidade de cuidado pela razão de que a família precisa ser orientada para 0 autocuidado e para ser em autônomas enão fiquena dependência dos profissionais da equipe (C1).

Tem sido esse o nosso caminho: abrir cada vez mais espaço para a família, para o contexto da família entendendo quea criança não édo hospital, não éda unidade E la vai para casa e é nessa casa, nessa família que el e será cuidado. D aí a necessidade de envolver as pessoas da casa no cuidado. O utro objetivo é conhecer as condi ções da casa, qual suportetem essa família para que a orientação da equipe de saúde não seja esquizofrênica (D 1).

São declarações que permitem compreender que o cuidado à criança deve ser dirigido para a família, entendida e percebida a partir de seu ambiente físico, social e cultural. Essa atitude vem permitindo aos profissionais compreensão ampliada do processo saúde/ doença e de intervenções que vão al ém das práticas curativas ${ }^{(11)}$.

Por outro lado, a aproximação com a família gera nos profissionais diferentes sentimentos que vão do estímulo e motivação para um trabal ho inovador ao sofrimento e apatia. Esta última nomeada como quietude dos cemitérios ${ }^{(12)}$ e definida como a reação de inércia resultante do desgaste do trabalhador de saúde por esse contato permanente com a dor, com o risco e o sofrimento em que vivem muitas das famílias brasileiras. As falas e os significados construídos denunciam estas assimetrias de sentimentos dos profissionais:

E ntrar na casa dessas famílias é constrangedor. É tanta pobreza... É uma situação que me incomoda, me dejxa desolada, triste. Volto pra a unidade com a certeza de que precisamos faz er muito. M e sinto estimulada a fazer. Não sei bem como, mas ando tentando. É preciso que ensinar essas famílias se indignarem com as condições em que moram, que vivem (P 8).

[ ...] o problema da criança éa doença e o da família é a completa falta de condições. E o quefazer? Como fazer? M esinto de pés e mãos atadas. Vivo lidando sempre com os mesmos problemas. A ndar por aqui, por esse bair ro é difícil. A qui falta tudo até dignidade. $\mathrm{E}$ pra isso faço quase nada (P 14).

Há dias que penso quenão sou capaz defazer nada ou quasenada por essas famílias, por essas crianças. Q uando o problema éa doença émais fácil agir. P rescreve se o tratamento, indica- seinternação, encaminha. M as há tantas outras coisas que remédio não resolve! 0 meu conhecimento médico fica pequeno demais (P22).

\section{D efendendo o preparo do profissional para atuar na ABS}

A formação de enfermeiros e médicos, apesar das propostas inovadoras nos currículos, a ênfase ainda se dá no espaço hospitalar, na doença e na cura, afastado muitas vezes do conhecimento oper ante. Por outro lado, a ABS espera receber um profissional capaz de dominar processos lógicos de construção dos saberes e práticas de modo coe rente com os aspectos sociais, epidemiológicos, econômicos e culturais em saúde. Estas são algumas das consider ações presentes nas falas dos participantes aqui recortadas nas dimensões da prática, do ensino e das perspectivas de mudanças decorrentes das exigências institucionais do cuidado em saúde e pelas experiências inovadoras do ensino superior:

[...] no momento em que existem profissionais despreparados para desenvolver as ações a integralidade perde 0 sentido, a filosofia da atenção perde 0 sentido, isso se di lui, se dissolve e o indivíduo passa a ser visto de forma fragmentado, estanque e setorizado. 0 ponto principal é o fortalecimento na formação de novas práticas que tem que partir da formação [ ...] épreciso investir na formação (D 1). 
A ABS deseja uma formação que não é de aquisição de técnicas, mas de habilidades para a relação com 0 outro - equipeefamília [ ...] são ações que exigem qualificação (D2).

As ciências da saúde são ciências do homem. As pessoas estão se preparando, se capacitando para cuidar de pessoas e não de doenças. 0 profissional de saúde não de ve preocupar-se com órgãos, mas preocupar-se com a pessoa [ ...] A academia desempenha um papel importante, não deformatar o caráter individual da pessoa, mas de agregar valores. E ssa éa questão pela qual estamos lutando (G 2).

São falas que nos remetem ao desafio de buscar e desenvolver novas competências profissionais. Cabe ressaltar que os profissionais devem ser formados aptos para o trabal ho técnico, mas também capazes de estabelecer estratégias que favoreçam o diálogo, a troca, a inter e a transdisciplinaridade e que efetivamente contribuam para as ações de promoção da saúde individual e coletivamente. Sugere ainda o resgate das questões humanitárias nas ações desenvolvidas pelos profissionais da saú$\mathrm{de}^{(13)}$.

Há movimentos em busca de transformações, tanto na organização dos cursos como das práticas pedagógicas em diferentes estágios de formuIação, acumulação e implementação nas diversas carreiras, regiões e contextos onde ocorrem os processos de formação dos profissionais de saúde ${ }^{(14)}$. Estes movimentos favorecem a construção de práticas cuidadoras coerentes com a integralidade. Favorecem novas atitudes e valores representados no discurso dos participantes da investigação:

F omos formados num cur rículo muito disciplinar, fragmentado, onde as profissões da área da saúde aprendiam a tratar doenças e o sujeito como um todo não era percebido. Fomos formados aprendendo a tratar a doença independente do indivíduo. D a mesma forma que se tratava um se tratava 0 outro. Começamos a perceber outro modelo de atenção que se diferencia em muito do quefoi a minha. Sefala de gente, de pessoas, de cuidado, apesar dos procedimentos, da doença e da cura ainda ser muito forte (D 3).

[ ...] ao entrar em contato com a comunidade 0 aluno começa a vivenciar e a perceber os problemas e, se forem sensíveis, via de regra as pessoas são sensíveis, passam a perceber os problemas da comunidade A idéa é fazer com que eles vivenciem isso no campo, que identifiquem as condições dessas famílias e problemati- zem. Assim eles poderão refletir, programar e plane jar intervenções que se aproximam da realidade dessas pessoas ( $\mathrm{C} 2$ ).

Há, portanto, uma preocupação em vincular a formação do profissional de saúde com os aspectos humanos e sociais com vínculo e responsabilização com a família e a comunidade. N esse sentido o papel das instituições educadoras na formação para o trabalho em saúde é mediar essa transição, permitindo aos estudantes vivenciar as situações e contextos e, a partir deles, tomar decisões em um movimento dialógico por onde circulam as dimensões técnicas, cognitivas, sociais e éticas do saber e do fazer na saúde e na doença.

\section{CONCLUSÕES}

M odelando a Integralidade do Cuidado na A BS é um processo no qual opera um jogo de expectativas e produções caracterizado como momento de construção singular e de atitudes cooperativas e solidárias para a prática do cuidado. É uma dimensão da integralidade que, como fenômeno social, ocorre em interação compreendendo que as respostas às necessidades da criança e da família nem sempre podem ser encontradas no setor saúde. E ssas constatações impõem uma série de desafios e inquietações que incluem pensar a saúde e o cuidado à criança como sistema complexo que se caracteriza, entre outras pretensões, pela integralidade do cuidado e pelas ações interdisciplinares e intersetoriais. Sob essa perspectiva as práticas de cuidado à criança, correspondem a um conjunto de ações, interações, e intervenções que objetivam a manutenção e a promoção da saúde para garantir o processo dinâmico do crescimento e do desenvolvimento infantil. Portanto, $M$ odelando a Integralidade do Cuidado na ABS requer romper com o instituído para re-criar novos modos de cuidados e de cuidar aliando ao saber técnico o saber operante e as tecnologias relacionais e subjetivas.

N esse sentido, a construção de novos conhecimentos e de práticas, na per spectiva da integralidade do cuidado, vem sendo uma constante preocupação e compromisso dos pesquisadores da Enfermagem, uma vez que tomam parte dessa prática, buscando avançar na contribuição de novos saberes e fazeres no cuidado de enfermagem e em saúde e nas políticas públicas do país. 


\section{REFERÊNCIAS}

$1 \mathrm{M}$ attos RA. Os sentidos da integ ralidade: al gumas reflexões acerca de valores que merecem ser defendidos. In: Pinheiro R, M attos RA, organizadores. Os sentidos da integralidade na atenção e no cuidado à saúde. Rio de Janeiro: IM S/ UERJ/ CEPESQ/ ABRASCO; 2006. p. 39-64.

2 Pinheiro R, Luz M T. Práticas eficazes $X$ modelos ideais: ação e pensamento na construção da integralidade. In: Pinheiro R, M attos RA, organizadores. Construção da integralidade: cotidiano, saberes e práticas em saúde. Rio de J aneiro: I M S/ U ERJ / CE PESQ / ABRASC0; 2003. p. 7-34.

3 Pinheiro R, Guizardi FL. Cuidado e integ ralidade: por uma geneal ogia de saber es e práticas no cotidiano. In: Pinheiro R, M attos RA, organizadores. Cuidado: as fronteiras da integralidade. Rio de J aneiro: CEPESC/ IM S/ UERJ/ABRASC 0; 2004. p. 21-36.

4 Figueiredo $G L$, M ello DF. A prática de enfermagem na atenção à saúde da criança em U nidade Básica de Saúde. Rev L atino-A m E nfermagem. 2004;11(4):54451.

5 Strauss AL, Corbin J. G rounded Theory M ethodology: an overview. In: D enzin N K, L incoln Y S. Handbook of quantitative research. T housand Oaks: Sage; 1994. p. 273-85.

6 Strauss AL, Corbin J. Pesquisa qualitativa: técnicas e procedimentos para o desenvolvimento de Teoria F undamentada. Porto Alegre: Artmed; 2008.

7 Sousa F G M . Tecendo a teia do cuidado à criança na A tenção Básica de Saúde: dos seus contornos ao en- contro com a integralidade [ tese] . F lorianópolis: U niver sidade Federal de Santa Catarina; 2008.

8 Schraiber LB, Peduzzi M , Sala A, N emes M IB, Kon R. Planejamento, gestão e avaliação em saúde: identificando problemas. Ciênc Saúde Colet. 1999;4(2):221-42.

9 M eirelles BHS, E rdmann AL . A interdisciplinaridade como construção do conhecimento em saúde e enfermagem. T exto Contexto Enferm. 2005;14(3):411-8.

10 N obre LC. T rabalho de crianças e adolescentes: os desafios da intersetorialidade e o papel do Sistema ú nico de Saúde. Ciênc Saúde Colet. 2003:8(4):96371.

11 M inistério da Saúde (BR ), Secretaria de Assistência à Saúde, Coordenação de Saúde da Comunidade. Saúde da F amília: uma estratégia para a reorientação do modelo assistencial. Brasília (DF ); 1998.

12 Campos RT O, Campos G W S. Co-construção de autonomia: o sujeito em questão. In: Campos GES, $M$ inayo M CS, A kerman M , D rumond J unior M , Carvalho Y M , organizadores. T ratado de saúde coletiva. São Paulo: H ucitec; 2008. p. 669-88.

13 A Imeida SG P, G er mano R M . A teoria da dádiva e o cuidar em enfermagem. Rev G aúcha E nferm. 2009; 30(2):338-42.

14 M acedo M CS, Romano RAT, Henriques RLM, Pinheiro R. Cenários de aprendizagem: intersecção entre os mundos do trabal ho e da formação. In: Pinheiro R, Ceccim RB, M attos RA, organizadores. Ensinar saúde: a integ ralidade e o SU S nos cursos de graduação na área da saúde. R io de Janeiro: I M S/ UERJ/ ABRASC0; 2006. p. 229-50.

\section{Endereço da autora / Dirección del autor / Author's address:}

F rancisca G eorgina M acedo de Sousa

Rua Viana Vaz, 230, Centro

65020-660, São Luís, M A

E-mail: fgeorginasousa@ hotmail.com
Recebido em: 25/ 04/ 2010

A provado em: 03/ 11/ 2010 